\title{
Theoretical and Experimental Investigation of the Dynamic Behaviour of Centrifugal Fans
}

\author{
G. MARIAUX*, Y. GERVAIS and L. CRESPEAU \\ Laboratoire d'Etudes Aérodynamiques, University of Poitiers, Batiment H, 40 Avenue du Recteur Pineau, \\ 86022 Poitiers Cedex, France
}

(Received 11 August 1997; In final form 17 September 1998)

\begin{abstract}
In this paper, a theoretical model is proposed to investigate the centrifugal fans' transient behaviour in response to sudden imposed changes in pressure or flow rate. We are especially interested in the dissipative or creative energy transient behaviour of the fans located in actual aeraulic networks.

The model is based on the global modelisation of the motor and resistant torques (due to pressure and viscosity actions on the blades) which are present on the rotor and by their coupling with its inertia.

The model is then validated by experimental measurements which show its validity. In the case of the tested fan, the instantaneous characteristics (in particular the flow rate and rotation speed) have been correctly predicted by the model, with regard to time as well as amplitudes, provided the flow is not reversed.
\end{abstract}

Keywords: Fan, Centrifugal, Unsteady, Behaviour, Validation, Energy

\section{INTRODUCTION}

The theoretical determination of the transient behaviour of fans subjected to instantaneous variations in high static pressure or flow rate is quite difficult due to the fan's constant time, related to the inertia of its rotor, which is usually greater than the constant establishment time of the change of pressure or flow rate. The result of this mechanical inertia is that the rotation speed of the rotor does not adapt instantaneously to the flow, and that the fan dissipates or creates more flow energy than it usually supplies. The use of the pressure-flow rate characteristics given by manufacturers for the steady state is then no longer possible.

We establish a theoretical model which takes into account the inertia of the fan's rotating parts from the fundamental dynamic relations applied to the rotating system. The motor and resistant torques on the blades - associated with pressure effects and friction losses - are written assuming quasistationary state for the fluid flow.

In order to calculate the transient behaviour of the fan, it is considered when operating in an

*Corresponding author. Tel.: (33) 549453809. Fax: (33) 549453663. E-mail: Gilles.Mariaux@brive.unilim.fr. 
aeraulic system, at a stable working point. It is then subjected to instantaneous changes of pressure or flow rate conditions. From the numerical resolution of the system composed of the fan and pipe unsteady behaviour equations, it is possible to obtain the instantaneous characteristics of the fan.

The experimental rig consists of a transient pressure generator connected to a duct containing a fan. The latter is associated to head losses in such a way as to obtain a stable working point. The pressure wave generator designed and constructed at the laboratory, allows the generation of pressure drops like Heaviside steps at the end of the pipe. Instantaneous pressure and rotation speed sensors and a hot wire anemometer are used to measure all the transient characteristics of the fan. The experimental results show that the theoretical model is quite accurate if the flow rate does not reverse.

\section{THEORETICAL MODEL}

\section{Dynamic Equation}

The instantaneous effects of variations of mean flow rate on the fan, considering its inertia, are given from the application of the fundamental dynamic relation applied to the rotating system:

$$
J \frac{\partial \Omega}{\partial t}=C_{\mathrm{m}}(\Omega)+C_{\mathrm{R}}(Q, \Omega)
$$

where $\Omega$ is the rotor rotation speed, $Q$ the volumic flow rate and $J$ the inertia of the total rotating mass. $C_{\mathrm{m}}(\Omega)$ is the motor torque and $C_{\mathrm{R}}(Q, \Omega)$ refers to the total resistant torques opposed to the rotor due to pressure and viscosity actions on the blades, as expressed by Mariaux (1995).

\section{Motor Torque}

The motor torque depends only on the rotation speed of the rotor $\Omega$. The most reliable representation of the instantaneous motor torque comes from the interpolation of the torque rotationspeed characteristic of the electric motor given by manufacturers.

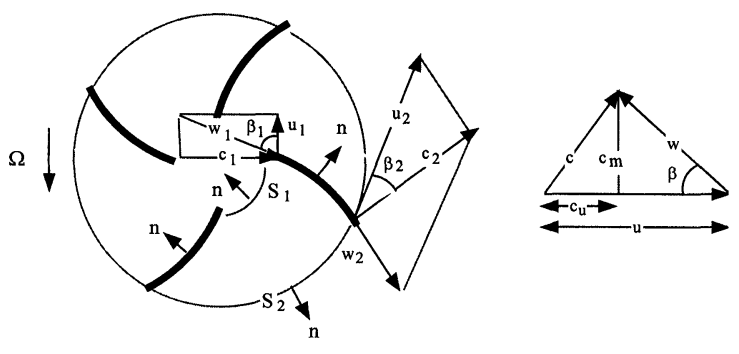

FIGURE 1 Composition of the fan impeller velocities (relative velocity $\boldsymbol{w}$, training velocity $\boldsymbol{u}$ and absolute velocity $\boldsymbol{c}$ ).

\section{Resistant Torque and Differential Pressure Generated}

The resistant torque depends on the instantaneous flow rate $Q$ and on the rotation speed $\Omega$. The resistant torque $C_{\mathrm{R}}(Q, \Omega)$ is defined by considering a succession of quasi-stationary states. In the case of the centrifugal fan, the resistant torque and the generated impeller pressure may be obtained from the application of the integral theorem of the moment of momentum over the volume enclosed by the blades, as shown by Comolet (1976) and Sédille (1973) (see Fig. 1).

\section{Theoretical torque and pressure generated}

In the case of air, gravity and unsteady effects due to fluid inertia can be neglected in relation to pressure effects and change of the moment of momentum as the control volume of the fan is very small. Therefore, the rotor torque due to pressure and viscosity on blades can easily be expressed by means of the distance $r$ from the running point to the rotor axis and impeller velocities at the same point. The relative velocity of the fluid with regard to the impeller is $\boldsymbol{w}$, the training velocity due to the impeller rotation at the velocity $\Omega$ is $\boldsymbol{u}$, and the absolute velocity is $c$. The resistant torque $C_{\mathrm{R}}$ is then obtained from the projection of the theorem of momentum on the fan rotation axis $\left(\boldsymbol{x}_{\nu}\right)$ and expressed by the the total flow rate $Q$, the radius $R$ and the projection of the absolute velocity on the orthoradial axis at impeller inlet $c_{u 1}$ and outlet $c_{u 2}$ which are assumed constant on $S_{1}$ and $S_{2}$ area. Its expression is described by 
Dick and Ammi (1991) as

$$
C_{\mathrm{R}}=-\rho_{0} Q\left(R_{2} c_{u 2}-R_{1} c_{u 1}\right)
$$

where

$$
c_{u 2}=u_{2}-c_{\mathrm{m} 2} \operatorname{cotan} \beta_{2}
$$

with

$$
u_{2}=R_{2} \Omega \quad \text { and } \quad c_{\mathrm{m} 2}=Q /\left(2 \pi R_{2} b_{2} \tau_{2}\right),
$$

$b_{2}$ being the depth of the blades extremities and $\tau_{2}$ an obstruction factor which takes into account the number of blades $(n)$, their thickness $(e)$ and their orientation. Since there is no cause of prerotation of the fluid in front of the blades, we have: $c_{u 1}=0$.

Applying the kinetic energy theorem to the smallest control volume enclosing the fan and neglecting impeller losses and unsteady effects due to fluid inertia, the whole power developed by the resistant torque at velocity $\Omega$ is released to the fluid and can be expressed as

$$
P_{\mathrm{f}}=C_{\mathrm{R}} \Omega=-\rho_{0} Q\left(\left(R_{2} \Omega\right) c_{u 2}\right)=-\Delta p \operatorname{th} Q
$$

where $\Delta p$ th is the theoretical differential pressure that could be obtained if there were effectively no losses.

\section{Correction of velocities}

Comolet (1976) shows that, as a result of the finite number of blades $(n)$ and due to Coriolis and centrifugal training strength, streamlines at the outlet of the impeller have a direction noticeably different from the one theoretically imposed by the blades (about $5^{\circ}$ to $10^{\circ}$ ). As described by Dick and Ammi (1991) and Wiesner (1967), theoretical torque and pressure drop are then slightly altered. The determination of the corrected $\left({ }^{\prime}\right)$ impeller characteristics needs the introduction of a slipping factor $(\sigma)$ or velocity $\left(c_{\mathrm{g}}\right)$ relative to training velocity $u$, which allows speed deflection to be taken into account. They are defined as follow:

$$
c_{\mathrm{g}}=c_{u 2}-c_{u 2}^{\prime}
$$

and

$$
\sigma=\left(u_{2}-c_{\mathrm{g}}\right) / u_{2}=\left(c_{u 2}^{\prime}+c_{m 2} / \tan \beta_{2}\right) / u_{2} .
$$

These two expressions were deduced from approximations of flow channel (enclosed by the blades) calculations taking into account the Coriolis and centrifugal driving strength:

$$
\sigma=1-\left(\sin \beta_{2}\right)^{1 / 2} / n^{0.7}
$$

\section{Pressure losses}

It is necessary to take into account the pressure losses associated with the flow through different parts of the fan: inlet, impeller, volute and diffusor. As described by Dick and Ammi (1991), these losses are related to viscous friction, singular head losses, or mismatching losses (shock) due to sudden changes of the moment of momentum which appear when the fan is not operating at its best efficiency point. These pressure losses have to be subtracted from the theoretical one expressed by Eq. (2) to obtain the effective power released to the fluid.

Pressure losses due to viscous friction are calculated as pipe friction and expressed as a fraction of the kinetic energy based on the flow rate $Q$. These pressure losses occur especially in the volute. Singular head losses occur in the fan inlet, upstream collision between flow and blades, and come from deflection between inlet fan flow and inlet impeller flow. They depend on the inlet geometry and its position with regard to the impeller. They are expressed as a fraction of the inlet kinetic energy based on the flow rate $Q$.

Mismatching losses arise firstly from the collision of the inlet flow when it encounters the impeller blades and secondly from the collision of outlet impeller flow and volute flow. They can be expressed as a fraction of the kinetic energy calculated from the tangential velocity component, respectively at the inlet $\left(c_{u 1}\right)$ and at the outlet $\left(c_{u 2}\right)$ of the impeller. These shock losses appear since operating conditions are different from those for which the fan was designed.

In the case of very low flow rate, the problems of representation of different phenomena are more complex due to two phenomena. The first one being that there is a flow circulation between each channel 
due to excess pressure on the fore-part of the blades and low pressure on the back. Secondly, there is a leakage flow rate between the casing and the impeller. Therefore, the present theory is invalid when these two phenomena are predominant with regard to flow rate $Q$, that is to say, for very low flow rates.

\section{Application and Resolution}

Let us now define the relation which gives the instantaneous speed of rotation of the fan resulting from flow rate variations. In order to investigate the dynamic response of the fan to sudden changes of pressure or flow rate, we have to consider the fan operating in a real aeraulic network, including head losses (see Fig. 2).

Applying the kinetic energy theorem to the domain defined by the above aeraulic network yields

$$
\begin{gathered}
\frac{\partial Q(t)}{\partial t}=\frac{S}{\rho_{0} L}\left[\frac{P_{\mathrm{f}}(Q(t), \Omega(t))}{Q(t)}+p_{\mathrm{r}}(t)-p_{\mathrm{a}}(t)\right. \\
\left.-\frac{1}{2} \rho_{0} \frac{\delta}{S^{2}}|Q(t)| Q(t)\right]
\end{gathered}
$$

where $P_{\mathrm{f}}$ is the power supplied to the fluid by the fan and the last term represents the dissipated power.

The dynamic behaviour of the fan may then be obtained from the first order differential system of Eqs. (1) and (3). The unknown values are the volumetric flow rate through the impeller (or the aeraulic circuit) and its speed of rotation, while time is variable. The system of equations is solved using the numerical Runge-Kutta method.

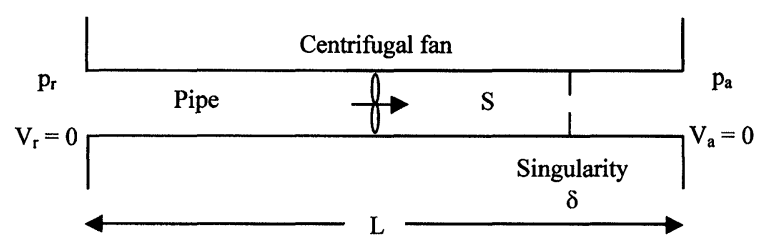

FIGURE 2 Aeraulic circuit enclosing the centrifugal fan.

\section{EXPERIMENTAL SET UP}

The experimental set-up designed by Mariaux (1995) is described in Fig. 2 and consists essentially of a centrifugal fan located in a pipe and loaded by a diaphragm in such a way that the working point has the same value as in simulations (see Fig. 3). The transient pressure generator, of very simple principle, is connected to the pipe inlet to generate pressure drops $p_{\mathrm{r}}(t)$. It consists of a large tank set at the required pressure by a fan and rapidly brought into contact with the pipe extremity by opening a valve driven by an actuator. It is then possible to change pressure drop intensity and establishment time. Two pressure piezzo-electric sensors are located just upstream and downstream of the fan (CP_1 and $\left.\mathrm{CP}_{-} 2\right)$. A hot wire anemometer (FC) measures flow velocity in the pipe while a tachometer measures the impeller rotational speed of the fan. The flow velocity allows the evaluation of the instantaneous flow rate.

\section{COMPARISON OF THEORETICAL AND EXPERIMENTAL RESULTS}

\section{Static Results}

Prior to any numerical simulation of the fan's transient behaviour, the model was first verified for its static characteristics. Therefore, by using this model, we can calculate the static characteristics (total differential pressure/flow rate and rotation speed/flow rate) of the fan which are then compared to experimental data points (see Figs. 4 and 5).

The theoretical and experimental pressures are in good harmony for most of the flow rate values.

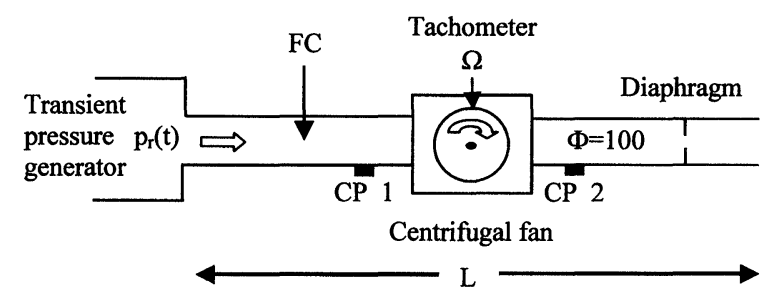

FIGURE 3 Simplified diagram of the experimental set-up. 
Evolution of pressure in terms of flow-rate

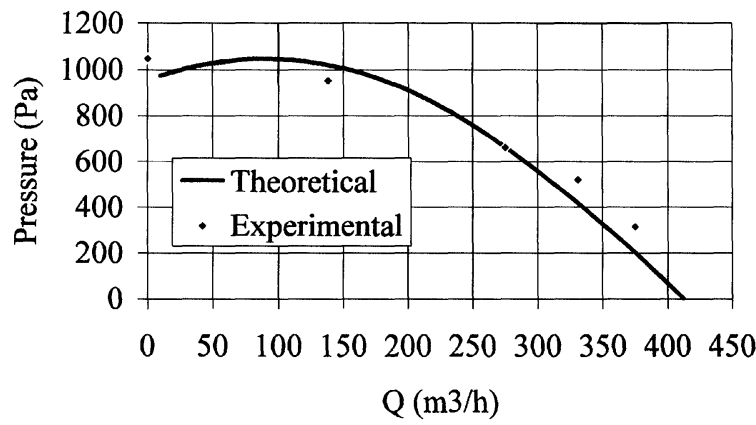

FIGURE 4 Comparison of the theoretical and experimental differential pressures generated by the fan in terms of flow rate and in steady state.

Evolution of rotation speed in terms of flow-rate

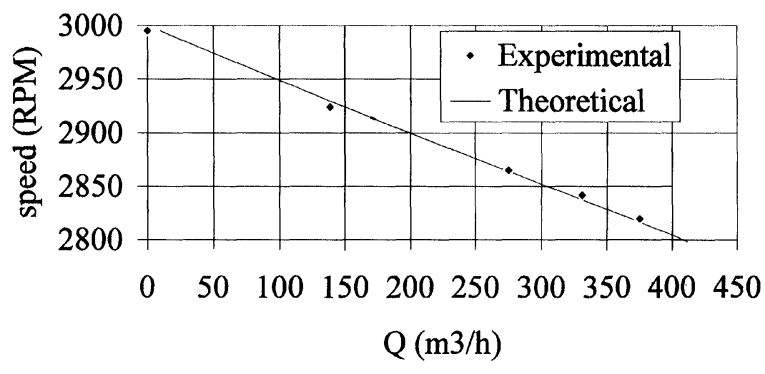

FIGURE 5 Comparison of the theoretical and experimental fan impeller rotation speeds in terms of flow rate and in steady state.

For flow rates between 200 and $300 \mathrm{~m}^{3} / \mathrm{h}$, the fan operates at its best efficiency point and hence the estimation given by the model is very accurate. Due to the appearance of the recirculation phenomenon of the fluid into the impeller, the model loses a part of its validity for low flow rates. For high flow rates, singular and friction losses are probably over-estimated. For the range from 75 to $200 \mathrm{~m}^{3} / \mathrm{h}$, the pressure losses due to the shocks on the blades and in the volute are prevalent and probably not very well represented.

The theoretical and experimental rotational speeds are in very good harmony and present a linear evolution in terms of flow rate. These last results show that the resistant torque $C_{\mathrm{R}}$ is correctly represented by the model.
Temporal evolution of pressure pr

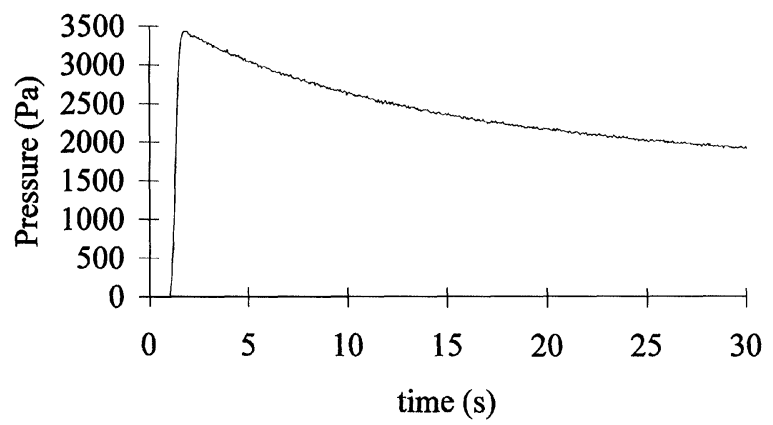

FIGURE 6 Temporal evolution of pressure $p_{\mathrm{r}}(t)$ imposed at the pipe extremity.

Temporal evolution of flow-rate

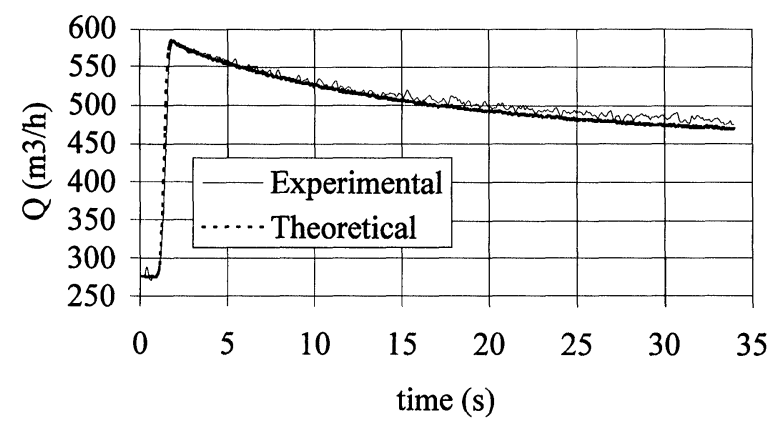

FIGURE 7 Temporal comparison of the theoretical and experimental volumetric flow rates in the pipe (or the fan) when it is subject to the pressure change $p_{\mathrm{r}}(t)$.

\section{Dynamic Response}

The fan is positioned in such a way that an increase of the pressure $p_{\mathrm{r}}(t)$ induces an increase of the flow rate. The pressure evolves as a pressure drop of intensity $3500 \mathrm{~Pa}$, characterized by a temporal variation rate reaching $10000 \mathrm{~Pa} / \mathrm{s}$ (see Fig. 6). Considering the functioning mode of the pressure wave generator and the flow rates involved, the pressure $p_{\mathrm{r}}(t)$ continues to evolve after the front wave.

\section{Instantaneous volumetric flow rate}

Figure 7 shows that the evolutions of the flow rate obtained from the model and experiments are entirely superimposable. In other words, the 
intensity variations and the constant time of the flow rate establishment are well represented.

\section{Instantaneous rotation speed of the impeller}

The remark about flow rate applies equally to the behaviour of impeller rotation speed, which is the same for theory and experiments, which can be seen in Fig. 8.

Instantaneous pressure generated

On the other hand, the comparison of the evolutions of the pressure generated by the fan provided by theory and experiments is less precise than that for the flow rate and rotation speed since the difference reaches $130 \mathrm{~Pa}$ which represents a relative difference of $13 \%$ (Fig. 9). This can be explained

\section{Temporal evolution of impeller rotation speed}

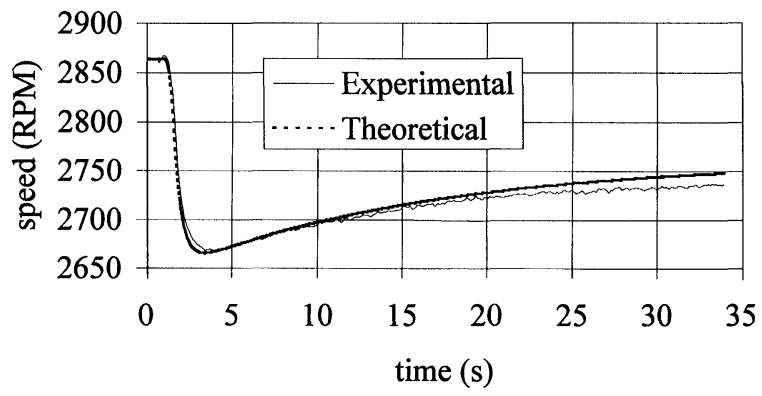

FIGURE 8 Temporal comparison of the theoretical and experimental impeller rotation speeds of the fan when the pipe is subject to the pressure change $p_{\mathrm{r}}(t)$.

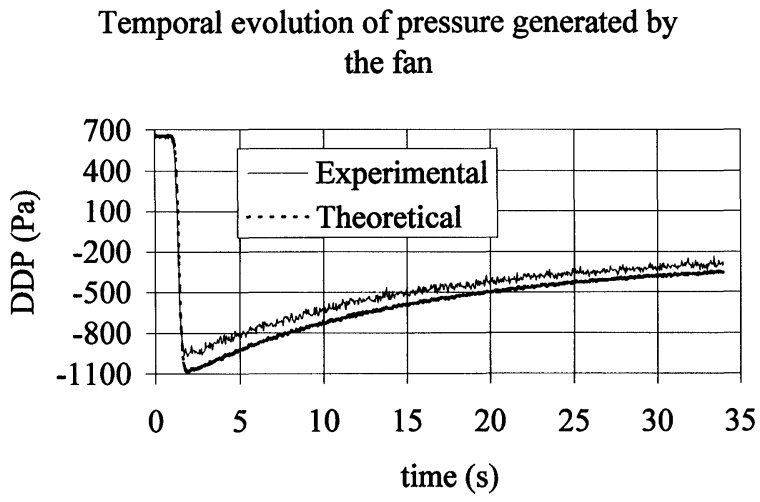

FIGURE 9 Temporal comparison of the theoretical and experimental pressure generated by the fan when the pipe is subject to the pressure change $p_{\mathrm{r}}(t)$. by the fact that the increase of the flow rate leads to an under-estimation of the pressure generated by the fan, in the case of the model. This result conforms with the static characteristics of the fan for which the pressure obtained from the model is under-evaluated compared to the experimental value obtained for high flow rates.

Note that the model is able to represent with accuracy the transient fan behaviour characterized by instantaneous flow rate, rotation speed and generated pressure even if the pipe is subjected to rapid variation of boundary conditions.

\section{CONCLUSIONS}

In this paper we have established a theoretical model to simulate the dynamic behaviour of a centrifugal fan in a real aeraulic network in response to instantaneous changes of flow rate. This model is based on the overall modelisation of the torques which are present on the rotor and by their coupling with its inertia. An experimental study shows that our simulation is quite accurate if the flow does not reverse in the pipe. The validated model presented is very interesting in order to study the dynamic behaviour of aeraulic networks including centrifugal fans.

\section{Acknowledgment}

The authors would like to thank Alstom Transport company for their financial support in these theoretical and experimental studies and Nicola Carmyllie for her help with translation.

\section{NOMENCLATURE}

$b_{1,2} \quad$ Depth of blades at impeller inlet and outlet (m)

c Absolute velocity $(\mathrm{m} / \mathrm{s})$

$c_{\mathrm{g}} \quad$ Slipping velocity $(\mathrm{m} / \mathrm{s})$

$C_{\mathrm{m}} \quad$ Motor torque $(\mathrm{Nm})$

$C_{\mathrm{R}} \quad$ Resistant torque $(\mathrm{N} \mathrm{m})$ 
$c_{u 1,2}$ Projection of absolute velocity on orthoradial axis at impeller inlet and outlet (m)

$e_{1,2} \quad$ Thickness of blades at impeller inlet and outlet (m)

$J \quad$ Rotor inertia $\left(\mathrm{kg} \mathrm{m}^{2}\right)$

$n \quad$ Number of blades

$p_{\mathrm{a}} \quad$ Ambient pressure (Pa)

$P_{\mathrm{f}} \quad$ Power supplied to the fluid (W)

$p_{\mathrm{r}} \quad$ Imposed pressure $(\mathrm{Pa})$

$Q \quad$ Volumetric flow rate $\left(\mathrm{m}^{3} / \mathrm{s}\right)$

$R_{1,2} \quad$ Radius at impeller inlet and outlet (m)

$S \quad$ Duct area $\left(\mathrm{m}^{2}\right)$

$t \quad$ Time (s)

$u_{1,2} \quad$ Training velocity at impeller inlet and outlet $(\mathrm{m} / \mathrm{s})$

$w \quad$ Relative velocity of the fluid with regard to the impeller $(\mathrm{m} / \mathrm{s})$

$\Omega \quad$ Impeller rotation speed ( $\mathrm{rad} / \mathrm{s})$

$\beta_{1,2} \quad$ Angle of blades with regard to training velocity at impeller inlet and outlet (rad) $\rho_{0} \quad$ Density $\left(\mathrm{kg} / \mathrm{m}^{3}\right)$

$\tau_{1,2} \quad$ Obstruction factor at impeller inlet and outlet (rad)

$\delta \quad$ Head losses coefficient of the duct singularity

$\sigma \quad$ Slipping factor

$\Delta p_{\text {th }} \quad$ Theoretical differential pressure $(\mathrm{Pa})$

\section{References}

Comolet, R. (1976) Mécanique expérimentale des fluides: Dynamique des fluides réels, turbomachines, Masson, Tome 2, $2^{\text {ème }}$ édition.

Dick, E. and Ammi, M. (1991) Comparison between steady and unsteady one-dimensional performance analysis methods for centrifugal pumps, European Journal, 36(3), 177-186.

Mariaux, G. (1995) Etude du comportement dynamique de circuits aérauliques: application au contrôle actif des variations rapides de pression dans les Trains á Grande Vitesse. Thesis $03 / 21 / 95$, Univeristy of Poitiers.

Sédille, M. (1973) Ventilateurs et Compresseurs Centrifuges et Axiaux, Aérodynamique générale, Calcul et fonctionnement, Eyrolles \& Masson, Tome 1.

Wiesner, F.J. (1967) A review of slip factors for centrifugal impellers, Transactions of the ASME, October 1967, 558-566. 


\section{ait \\ ENERGY MATERIALS}

M A N E Y publishing

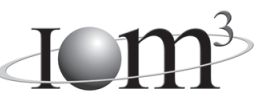

\section{Materials Science \& Engineering for Energy Systems}

Maney Publishing on behalf of the Institute of Materials, Minerals and Mining

The Institute of Materials, Minerals \& Mining

Economic and environmental factors are creating ever greater pressures for the efficient generation, transmission and use of energy. Materials developments are crucial to progress in all these areas: to innovation in design; to extending lifetime and maintenance intervals; and to successful operation in more demanding environments. Drawing together the broad community with interests in these areas, Energy Materials addresses materials needs in future energy generation, transmission, utilisation, conservation and storage. The journal covers thermal generation and gas turbines; renewable power (wind, wave, tidal, hydro, solar and geothermal); fuel cells (low and high temperature); materials issues relevant to biomass and biotechnology; nuclear power generation (fission and fusion); hydrogen generation and storage in the context of the 'hydrogen economy'; and the transmission and storage of the energy produced.

As well as publishing high-quality peer-reviewed research, Energy Materials promotes discussion of issues common to all sectors, through commissioned reviews and commentaries. The journal includes coverage of energy economics and policy, and broader social issues, since the political and legislative context influence research and investment decisions.

\section{CALL FOR PAPERS}

Contributions to the journal should be submitted online at http://ema.edmgr.com

To view the Notes for Contributors please visit: www.maney.co.uk/journals/notes/ema

Upon publication in 2006, this journal will be available via the Ingenta Connect journals service. To view free sample content online visit: www.ingentaconnect.com/content/maney

For further information please contact:

Maney Publishing UK

Tel: +44 (0)113 2497481 Fax: +44 (0)1132486983 Email: subscriptions@maney.co.uk

or

Maney Publishing North America

Tel (toll free): 8662975154 Fax: 6173546875 Email: maney@maneyusa.com

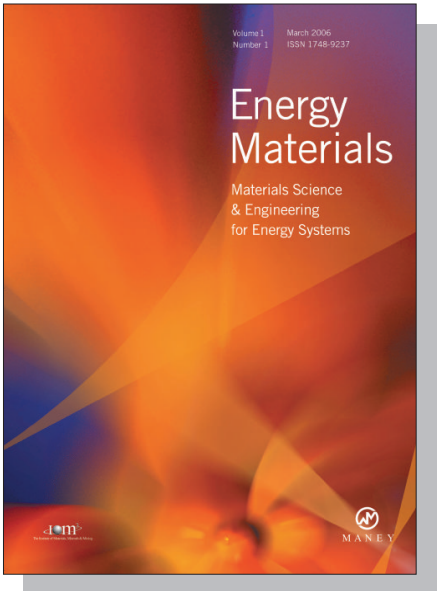

EDITORS

Dr Fujio Abe

NIMS, Japan

Dr John Hald, IPL-MPT, Technical University of Denmark, Denmark

Dr R Viswanathan, EPRI, USA

\section{SUBSCRIPTION INFORMATION}

Volume 1 (2006), 4 issues per year

Print ISSN: 1748-9237 Online ISSN: 1748-9245

Individual rate: $£ 76.00 / U S \$ 141.00$

Institutional rate: $£ 235.00 /$ US $\$ 435.00$

Online-only institutional rate: $£ 199.00 / U S \$ 367.00$

For special $\mathrm{IOM}^{3}$ member rates please email

subscriptions@maney.co.uk

\section{For further information or to subscribe online please visit www.maney.co.uk}



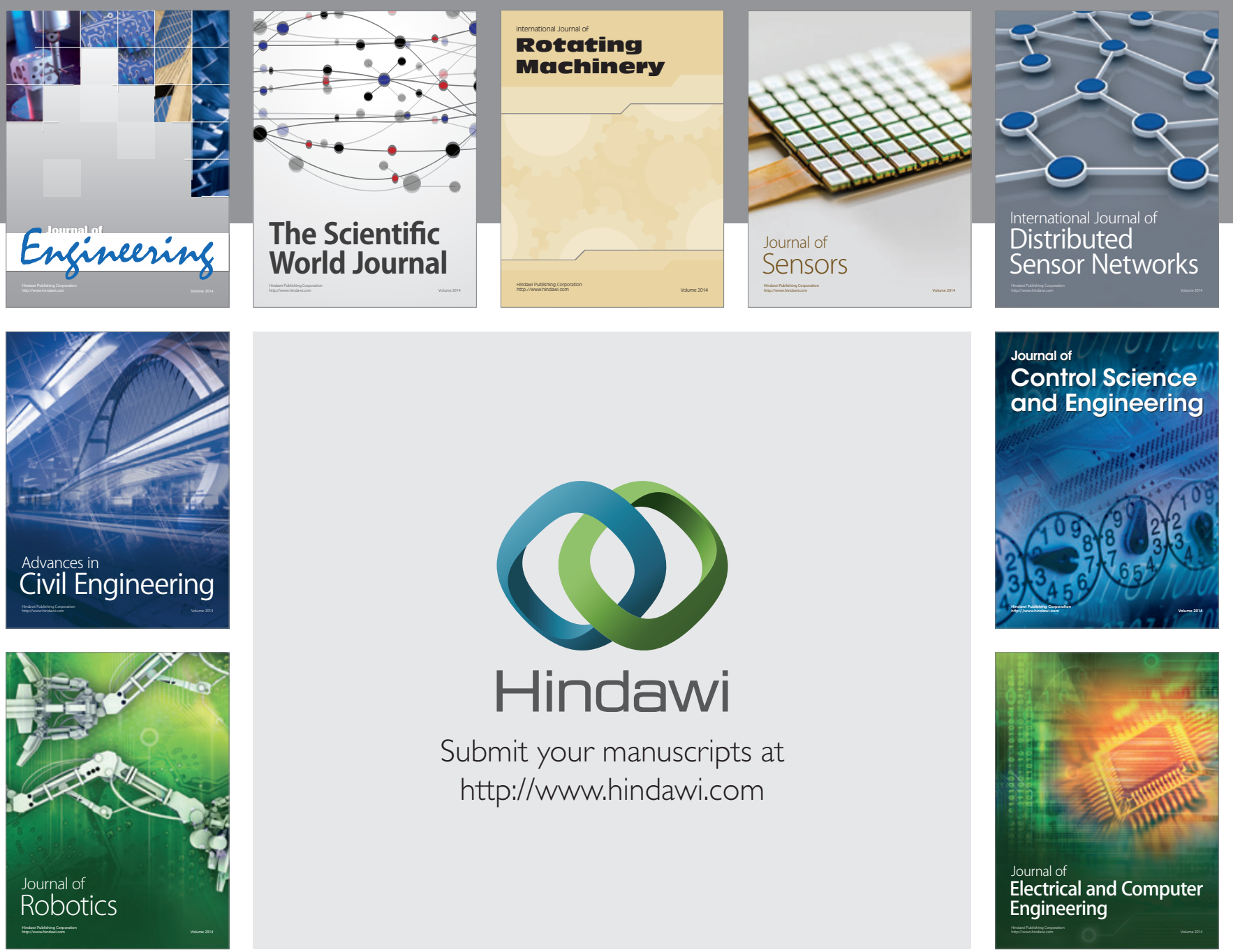

Submit your manuscripts at

http://www.hindawi.com
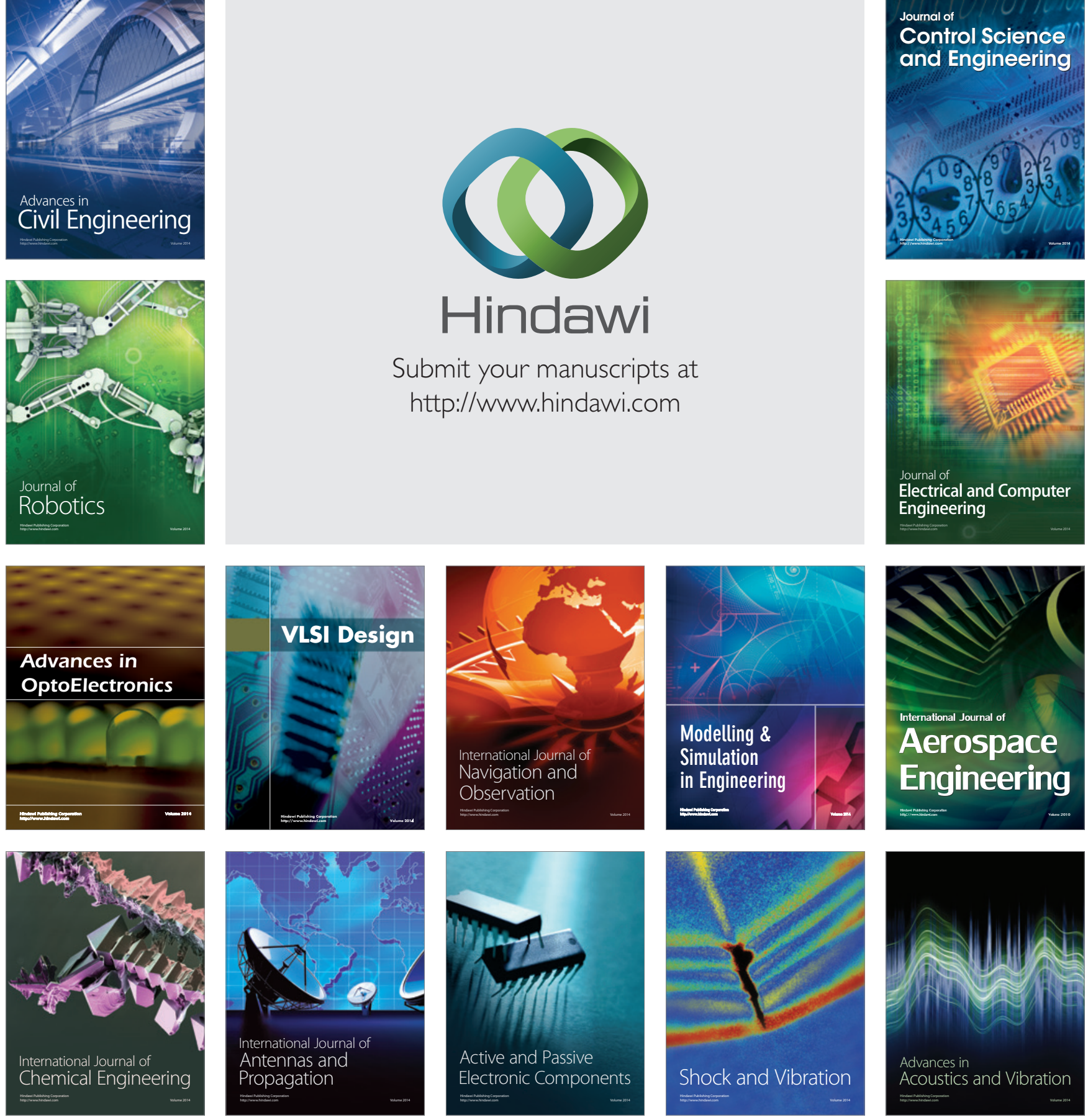